\title{
LA DIRECCIÓN NACIONAL DE MIGRACIONES ENTRE 2003 Y 2015: ACCIONES PARA EL CAMBIO DE CULTURA ORGANIZACIONAL. UNA MIRADA “DESDE ADENTRO”
}

\author{
The Dirección Nacional de Migraciones between 2003 and 2015: \\ actions for the change of organizational culture. \\ A look "from the inside"
}

María Dolores Linares*

\begin{abstract}
Resumen. La Dirección Nacional de Migraciones (DNM) es el organismo estatal de aplicación de la política migratoria en la República Argentina. A partir de la sanción de la Ley $N^{\circ} 25.871$ (2003) el funcionamiento de la DNM fue evaluado y modificado, generando reacciones al interior del mismo. Nuestro objetivo es identificar los principales cambios institucionales realizados a fin de adecuarse a la nueva normativa y examinarlos a partir de la perspectiva de sus funcionarios y empleados. Mediante la revisión de la bibliografía especializada y de un cuerpo normativo seleccionado, se procedió luego con un abordaje metodológico cualitativo basado en la realización de entrevistas a miembros de la DNM de distintas delegaciones analizando dos variables: el funcionamiento de la DNM previo al 2004 y las resistencias a los cambios al interior del organismo.
\end{abstract}

Palabras clave: instituciones; política pública migratoria; Dirección Nacional de Migraciones; Argentina.

\begin{abstract}
The Dirección Nacional de Migraciones (DNM) is the state agency in charge of applying the migration policy in Argentina. Since the enactment of the Immigraton Law N 25.871 of 2003, the DNM's operation was evaluated and modified, generating different reactions within. This article proposes to identify the institutional changes carried out in this agency to adapt to the new law institutionally, examining them from the perspective of its employees. Methodologically, we analyzed the content of specialized bibliography and legislation and we proceeded with a qualitative methodological approach by conducting interviews with employees and former employees of the DNM from different Delegations analyzing two variables: the functioning before the year 2004 and the resistances to the changes inside the institution.
\end{abstract}

Keywords: institutions; public immigration policy; Dirección Nacional de Migraciones; Argentina.

Investigadora del Consejo Nacional de Investigaciones Científicas y Tecnológicas (CONICET) Instituto de Estudios Socio-Históricos (UNLPam). Santa Rosa, La Pampa, Argentina. 


\section{Introducción}

La Dirección Nacional de Migraciones (en adelante DNM) es, desde 1949, el organismo del Estado dependiente del Ministerio del Interior y de Transporte (desde 2016 denominado Ministerio del Interior, Obras Públicas y Vivienda), encargado de aplicar la política migratoria en toda la República Argentina. Tiene una estructura organizativa territorial compuesta por una Sede Central y un Centro de Documentación Rápida en la Ciudad Autónoma de Buenos Aires, 29 Delegaciones en todo el país, seis Oficinas Migratorias y cuatro unidades móviles de documentación y trámites. La función, misión, visión y prioridades de la DNM quedaron determinadas por la Ley de Migraciones $N^{\circ} 25.871$ del año 2004 (aprobada en diciembre del 2003 y publicada en el Boletín Oficial en enero del 2004) y su Decreto Reglamentario 616/2010, según las cuales la DNM tiene la "competencia" para: entender en la admisión y otorgamiento de residencias; conceder permisos de ingresos, prórrogas de permanencia y cambios de calificación para extranjeros; controlar el ingreso y egreso de personas al país; ejercer el control de permanencia y el "poder de policía de extranjeros en todo el Territorio de la República"1 (Art. 107 de la Ley 25.871).

La sanción de la Ley N² 25.871 del año 2004 significó, según algunos investigadores y actores sociales (cf. Courtis, 2006; Novick, 2008; Nejamkis, 2016; CELS, 2005, entre otros), un giro en la política migratoria argentina en relación a la ley anterior, la Ley 22.439 del Poder Ejecutivo Nacional (en adelante PEN), Ley General de Migraciones y de Fomento de la Inmigración, conocida como Ley Videla de $1981^{2}$ - al incorporar un enfoque regionalista basado en los derechos de los migrantes y en el derecho humano a migrar. Este giro puso en evidencia que la DNM no podía cumplir su función sin un cambio sustantivo en su estructura, en sus objetivos y en su cultura organizacional. Para lograr la cristalización institucional se llevaron a cabo acciones al interior de la DNM que respondieron a variados objetivos y que fueron comprendidas de diferentes maneras al interior del organismo. En este artículo nos proponemos identificar dichas acciones y analizarlas desde la perspectiva de los funcionarios y empleados del propio organismo estatal según dos ejes: el funcionamiento de la institución previo al año 2004 y las resistencias encontradas u observadas.

Para lograr nuestro objetivo realizamos un análisis de contenidos mediante la revisión de la bibliografía especializada y de un cuerpo compuesto por leyes, decretos del PEN y normativa interna de la DNM (resoluciones y disposiciones) destinados a evidenciar la cristalización institucional del

\footnotetext{
1 Es importante notar que este artículo no contiene cambios sustanciales con respecto a la normativa anterior (Ley 22.439 de 1981).

2 La orientación de la Ley Videla fue principalmente restrictiva y prevaleció una óptica de seguridad policial en la regulación de la política migratoria.
} 
cambio normativo. Por otro lado, se procedió con un abordaje metodológico cualitativo para la generación de fuentes primarias mediante la realización de entrevistas a cinco (5) funcionarios y ex funcionarios, empleados y ex empleados $^{3}$ de la DNM de distintas delegaciones (Sede Central, La Pampa y Rosario). Todas las entrevistas fueron presenciales, realizadas sobre la base de un cuestionario que constaba de cinco preguntas abiertas, idénticas para todos los entrevistados y grabadas digitalmente ${ }^{4}$. Las primeras entrevistas se realizaron en la Delegación La Pampa en los años 2014 y 2015 en el marco de un proyecto de investigación grupal más amplio ${ }^{5}$ y luego, mediante la técnica de "bola de nieve" se establecieron los contactos con otros empleados y ex empleados para realizar las entrevistas en Rosario y Buenos Aires en el año 2016, para lo cual se viajó especialmente a dichas ciudades ${ }^{6}$. Con el fin de garantizar su anonimato, los entrevistados serán referenciados como DNM $\mathrm{N}^{\circ} 1, \mathrm{DNM}^{\circ} \mathrm{N}^{\circ} 2$ y así sucesivamente ${ }^{7}$.

Este artículo se organiza de la siguiente manera: en primer lugar se definen los lineamientos conceptuales y metodológicos sobre los cuales se basa nuestro análisis. Posteriormente repasaremos los objetivos y acciones

3 Cuando utilizamos el término "empleados/as" hacemos referencia a aquellas personas que tienen cargos de bajo rango dentro de la DNM, que forman parte de la "planta permanente" de la institución y que pueden haber realizado un recorrido laboral por varios departamentos o direcciones dentro de la misma. Los "funcionarios", por otra parte, detentan un alto cargo directivo que, en todos los casos, fue promovido por una decisión política. Algunos funcionarios entran a la DNM sin antes haber tenido un cargo de empleado (como los casos de los Directores/ as o Sub-Directores/as Generales). Otros, como dos de nuestros entrevistados, tuvieron un cargo de funcionario (Director/a de Delegación) luego de haber sido empleados por más de 10 años dentro de la DNM. Como veremos, el tipo de cargo detentado influye en la lectura y apreciación de los cambios realizados al interior del organismo.

4 Luego de realizar las desgrabaciones de las entrevistas, se procedió a la sistematización de información priorizando tres aspectos: la información aportada, las sensaciones expresadas y los términos comunes dentro del "lenguaje nativo" institucional. Buscamos acercarnos no tanto a la estructura del discurso de los entrevistados sino, como advierte Arfuch, también de "lo ausente, lo contradictorio, lo que se dice sin querer decir" (Arfuch, 1987, p. 48). El análisis del discurso aquí utilizado focaliza en la palabra y en los silencios, las risas y las muecas de disgusto de los entrevistados, atravesado por el contexto en el cual la palabra se dice. De esta manera intentamos corroborar el primer aspecto analítico (la información basada en el recuerdo y atravesada por la sensación) con los datos recuperados de la bibliografía y la legislación, enriqueciéndolo con los segundos dos aspectos. Estimamos que a partir de este cruce pudimos dar cuenta de los ejes sensibles del cambio institucional.

5 Se trató de un Proyecto Orientado a la Investigación Regional (POIRe) del año 2013, aprobado por Res.158/13 y 344/13 del CS de la Universidad Nacional de La Pampa y dirigido por la Dra. Andrea Lluch. El proyecto se titulaba "Instituciones, procesos y políticas del desarrollo económico-social en La Pampa: un enfoque interdisciplinario y de largo plazo".

6 Otras entrevistas se realizaron en la cuidad de Posadas, Provincia de Misiones durante el 2017 pero no fueron incluidas en este artículo.

7 A continuación de las referencias al final del artículo, se colocan los perfiles de los entrevistados según lugar de delegación, tipo de cargo, antigüedad, año en que fue realizada la entrevista y si continúa o no en el cargo. 
institucionales más relevantes de la Dirección Nacional de Migraciones entre 2004 y 2015. En tercer lugar describiremos cómo fueron comprendidas estas acciones "desde adentro" del organismo, a partir de los resultados de las entrevistas realizadas. El artículo concluye retomando las ideas principales y proponiendo líneas para profundizar el tema de investigación.

\section{El Estado: más allá de los agentes y las instituciones}

Frente al interés de analizar los cambios producidos en el seno de un organismo del Estado a raíz de una modificación en la política pública migratoria nos preguntamos, en primer lugar, desde qué óptica abordar al Estado. En un primer momento, la perspectiva institucionalista de la ciencia política nos brindó algunas pistas para comprender el complejo entramado de instituciones al interior del Estado (Linares, 2017). Tomamos el concepto de Evans (2007) quien entiende a las instituciones en tanto "reglas del juego" definidas históricamente y construidas socialmente. Este conjunto de reglas puede ser formal e informal (Acuña, Chudnovsky, 2013, p. 54) y estaría destinado a resolver problemas de coordinación y conflictos distribuidos en una sociedad. Esta postura permite vislumbrar al cambio institucional como una forma de administrar y resolver conflictos y no como una anomalía disruptiva.

En cuanto al Estado y a las políticas públicas, entendemos al primero siguiendo a O'Donnell y Wolfson (1993) quienes, aún con una marcada impronta weberiana, lo describen como una entidad que va más allá del aparato estatal o del conjunto de las burocracias públicas. Para los autores, el Estado es un conjunto de relaciones sociales que establece un orden social en un territorio determinado, respaldando dicho orden con una garantía coercitiva centralizada (ibidem, p. 165). Por otro lado, Skocpol (1995) establece que los Estados tienen la capacidad para fijarse objetivos y llevarlos a cabo mediante la producción de políticas públicas. Las políticas públicas son, según Oszlak y O’Donnell (2007), un conjunto de acciones y omisiones que manifiestan una modalidad de intervención del Estado en relación a una cuestión que concilia el interés de otros actores de la sociedad civil. Las políticas públicas migratorias, que forman parte de la política de población, serían las propuestas y metas elaboradas desde el aparato estatal con el fin específico de influir en el tamaño, la composición, el origen, la dirección, el asentamiento y la integración de los flujos migratorios espontáneos o pertenecientes al proceso global de planificación económico-social (Mármora, 1988, apud Nejamkis, 2016).

Ahora bien, aunque esta perspectiva - presentada de manera algo esquemática- se presente como "Estado-céntrica", nuestra intención no es atribuirle al Estado nacional un poder impersonal, legal y normalizador que, mediante sus prácticas "legales y legítimas" llegue de manera unívoca 
e indiscutida a todo el ámbito de lo social. Tenemos presente los riesgos de apoyarnos en una visión normativa del Estado que nos lleve a "pensar desde el Estado" y caer en un "reduccionismo institucionalista" (Espinoza Molina, 2015) o en una reificación del Estado. El Estado ni "está en todos lados" ni "es sólo una ficción" (Bourdieu, 1996, apud Schavelzon, 2010), sino que, como plantea Abrams (1988) tiene por un lado una base material y otra ilusoria. Se advierte así sobre lo "secreto" en el poder estatal: "(...) el Estado, como entidad autónoma y distinta, (...) es realmente poderosa y un aspecto de su poderío es su capacidad para impedir el estudio adecuado del Estado" (ibidem, p. 83). Para este autor el Estado es ante nada una ilusión que esconde un sistema de poder y dominación sumamente material, plausible de ser desentrañado para acceder a la verdadera práctica política. Pero no sólo se trata de secretos, acercarse a una institución estatal implica también dar cuenta de la diferencia entre la "ley" (las normas que rigen dicha institución, por ejemplo) y la "realidad" de lo que sucede al interior de las mismas (Leal, 2006). Especialmente en un caso como el nuestro donde la actividad burocrática no siempre siguió los procedimientos "normales" sino que se basó en la discrecionalidad de funcionarios y empleados, según veremos en las entrevistas realizadas.

Entonces, para echar luz sobre la "oscuridad" a la que apelan algunos de nuestros entrevistados al describir a la DNM, el método etnográfico que propone la antropología del Estado puede ser de utilidad para acercarnos a las dos caras de la institución estatal, tanto la material como la simbólica. Este abordaje implica, en primer lugar, desmitificar al Estado de su investidura de aparato homogéneo de dominación central (Corrigan, Sayer, 1985) y relevar su construcción por parte de actores diversos, en este caso, los funcionarios y empleados. Invertimos entonces la escala, complementando la búsqueda normativa con las entrevistas en profundidad a los empleados de la DNM. Cambiar la escala no significa oponer un "arriba" sobre un "abajo", sino reconocer -y utilizar como herramienta heurística, como indica Revel (2015)que "una realidad social no es la misma según el nivel de análisis o [...] la escala de observación en la que decidamos ubicarnos" (ibidem, p. 15). Y por otro lado, además de un cambio de escala necesitamos corrernos de lugar de abordaje para observar el "Estado desde adentro", como indican Bohoslavsky y Soprano (2010), buscando comprender justamente la dinámica organizacional e institucional de las distintas agencias del Estado. Entre la premisa de "no personalizar al Estado" (ibidem) y la de "personalizar al estado" definiendo "quién y quiénes son el Estado en cada momento determinado", intentaremos descifrar si la pretendida "impersonalidad" del Estado estaría ausente o no en la agencia de los cuadros políticos y administrativos de la DNM entre los años 2004 y 2015. 


\section{Los objetivos y acciones de la Dirección Nacional de Migraciones entre 2004 y 2015: los desafíos del cambio de orientación}

Antes de la reglamentación de la Ley 25.871 en el año 2010, la DNM utilizaba para su funcionamiento interno el Reglamento de Migración de 1965 según el Decreto PEN 4418/65 y contaba con una estructura organizativa que databa del año 1996, determinada por el Decreto del PEN 1659/96, acorde al espíritu de la normativa anterior. Debido a estos desajustes entre la "ley" y la "realidad" del organismo, en el mes de julio de 2004 el Decreto 836 del PEN declaró su emergencia administrativa porque -entre otros motivos como la detección de redes delictuales dedicadas al tráfico de personas- la institución presentaba "severas irregularidades operativas" así como fallas en la estructura organizativa y en la dotación del personal.

El decreto anunciaba que se había realizado una evaluación de la situación y los principales problemas en cada área de la DNM, que figuran en el Informe de Control Interno y Gestión (ICIG) del período 2003-2007 tienen que ver con a) la ausencia de mecanismos acordes a la dinámica de la gestión en todas las áreas (sistema contable y presupuestario, inmigrantes indocumentados por procesos arbitrarios y confusos, sistema informático obsoleto, etcétera); b) deficiencias edilicias y de infraestructura (oficinas, delegaciones y puestos de control); c) existencia de "normativa migratoria antigua totalitaria" y d) la falta de información y asesoramiento a migrantes.

Los objetivos y acciones que se dio el Estado a través de la DNM para solucionar los problemas mencionados se detallan en los Informes y Memorias Institucionales de Gestión, que figuran en la página web del organismo divididos en dos tipos: el Informe de Control Interno y Gestión (ICIG) antes citado, que abarca el período junio del 2003 hasta junio del 2007 y que corresponde a la dirección Ricardo Rodríguez (2003-2007), y ocho (8) Memorias Institucionales de Gestión para cada año entre 2008 y 2015 que corresponden a la administración de Martín Arias Duval (2007-2015).

En el Informe de Control Interno y Gestión (ICIG) (2003-2007) se plantean como objetivos procurar un marco normativo para la regularización de todos los inmigrantes "a fin de permitir su inserción legal y social" y asegurar los controles sobre las personas en el ingreso y egreso al país, respondiendo así a las funciones básicas de la DNM según algunos de nuestros entrevistados: el control, tanto de permanencia como de las fronteras. Pero en las Memorias Institucionales de Gestión (MIG) del 2008 se agregaron otros objetivos que buscaban adecuarse a la nueva normativa así como a un nuevo estilo de gestión pública basado en la eficiencia (que buscaban cumplir con los compromisos de calidad asumidos en la Cuarta Carta Compromiso con el Ciudadano y en las Normas ISO 9001: 2008). Estos nuevos objetivos fueron el de "intensificar 
la política migratoria inclusiva"; "diseñar y aplicar programas para la mejor gestión de funciones y servicios"; "reglamentar la Ley 25.871"; "mejorar la imagen institucional web"; "lograr mayor federalismo a través de las reuniones de delegaciones" y "gestionar una nueva estructura organizacional". En el año 2010 se suma un objetivo que será central en nuestro análisis: lograr el "cambio de cultura organizacional". Y en el año 2011 se redacta un objetivo general que sintetizaría la nueva orientación de la gestión:

Ser un organismo ágil, moderno, eficiente y transparente. Comprometido con la plena integración de las personas y el respeto a los derechos humanos del migrante. Generador de información útil para la toma de decisiones en materia demográfica o poblacional, y para la cooperación inter-jurisdiccional e internacional. (MIG, 2011, resaltado de la autora)

Observamos que se incorporan aquí cuestiones vinculadas a las capacidades de gestión del Estado, que analizamos en otra oportunidad a partir de la perspectiva de las capacidades estatales (Linares, 2017). Además, a partir de ese año se explicitan algunos objetivos tendientes a alcanzar el "cambio de cultura organizacional": profundizar los vínculos con las colectividades de migrantes y constituir un ámbito académico para "perfeccionar el conocimiento, actitudes y habilidades de los agentes gubernamentales, no gubernamentales y la sociedad civil en general, vinculados al ámbito migratorio y de refugiados" (MIG, 2012, resaltado de la autora).

En cuanto a las acciones tendientes a alcanzar estos objetivos, las sintetizamos según cuatro ejes ${ }^{8}$ :

1- legislación: Creación de Comisión Asesora, reglamentación de la Ley (Decreto 616/2010), creación del Manual de Procedimientos de Control del Movimiento Migratorio, Programa de regularización para dominicanos y senegaleses (Dis. DNM № 1/13 y 2/13) y Programa Siria, entre otras.

2- cambios organizacionales y apertura de delegaciones: firma de la Carta Compromiso Ciudadano, diseño tecnológico del Sistema informático de Admisión de Extranjeros (SADEX), modernización web, Turnos online, reestructuración del organigrama institucional, Instrumentación firma y expediente digital, cursos de Capacitación para empleados y funcionarios, creación de Mesa de Diálogo Migratorio para analizar y mejorar las políticas públicas y apertura de Delegaciones en San Luis, Alte. Brown, Florencio Varela y Villa Gesell, entre las acciones más destacadas.

3- apertura institucional y cooperación interorganizacional: Campaña gráfica institucional, actividades culturales con colectividades, cooperación con el Plan "Ahí" del Ministerio de Desarrollo Social, asistencia y asesoramiento con los Centros de Acceso a la Justicia (CAJ), acuerdo entre la DNM, la CGT, la Universidad de Tres de Febrero, la Organización Internacional del Trabajo, la OIM y el IPMA.

\footnotetext{
8 Para una descripción más completa y detallada de estas acciones, ver Linares, 2017.
} 
4- controles de permanencia y fronterizos: Aumento operativos de control de permanencia (1.454 en 2009, 1.915 en 2010, 3.109 en 2011, 3.034 en 2012, 4.000 en 2013, 3.933 en 2014 y 4.300 en 2015) y contratación y capacitación de personal para pasos fronterizos.

A partir de la revisión de las fuentes consultadas observamos que la mayoría de las acciones institucionales "novedosas" de DNM estuvieron centradas en la adecuación normativa y a instalar una nueva forma de gestión. Específicamente, se focalizaron en el aumento de personal (que pasó de 653 en 1996, a 1.708 en 2008, 2.084 en 2012 y más de 3.000 empleados en 2015) ${ }^{9}$, la capacitación, el mejoramiento de los recursos informáticos y tecnológicos del organismo, el mejoramiento en la atención al migrante (turnos online, eliminación de gestores, folletería explicativa) y los cambios en la estructura. El objetivo del "cambio de cultura organizacional" se buscó a partir de las acciones tendientes a cambiar las mentalidades de los empleados a partir de acciones concretas, algunas directas como los protocolos de atención, otorgamiento de turnos y capacitación y otras indirectas como los vínculos con colectivos de migrantes, ONGs y centros académicos o la participación en las fiestas de inmigrantes. Es importante destacar que, mientras estas acciones "novedosas" tendían a garantizar el respeto de los DDHH del migrante y la mejora de la atención, a la par la DNM aplicaba asimismo nuevas tecnologías destinadas a sus acciones más "clásicas", es decir, orientadas a mejorar los dispositivos de control, tanto migratorios como de permanencia: estos últimos aumentaron considerablemente en este período: mientras en el año 2009 se realizaron 1.454, en el 2015 Ilegaron a efectuarse 4.300 operativos de control de permanencia.

\section{Cambio institucional: los desafíos de la DNM "desde adentro"}

Hasta aquí hemos indicado de qué manera un organismo del Estado se propuso objetivos y acciones a fin de llevar a cabo de manera eficiente una nueva política pública. La normativa, las disposiciones, la "ley" y el Estado se nos presentan por el momento como un todo homogéneo con capacidad para cumplir con dichos objetivos sin fisuras. Sin embargo, en las entrevistas a funcionarios, exfuncionarios, empleados y exempleados de la DNM notamos tensiones y desajustes que, puestas en juego unas con otras, evidencian disputas y formas de pensar a la cuestión migratoria que se contradicen y excluyen mutuamente.

Las acciones ya enunciadas, pero sobre todo las destinadas a los cambios de la cultura organizacional, marcaron un "antes y un después" al interior de la DNM según nuestros entrevistados, que fueron evaluando la direccionalidad

\footnotetext{
9 Según datos proporcionados por el Decreto 1659/96 del P E.N., la Decisión Administrativa 250/2008, J.G.M., la Decisión Administrativa 1395/2012, J.G.M. y la MIG del año 2015.
} 
de los cambios según sus experiencias personales con la institución. Nos propusimos considerar estas percepciones a partir de dos ejes que pudieran dar cuenta de ese momento de transición entre el "pre 2004" y el "post 2004": el funcionamiento de la DNM previo al año 2004 y las resistencias a los cambios encontradas u observadas dentro del organismo.

\section{La DNM antes de la Ley $\mathbf{2 5 . 8 7 1}$}

Sobre la experiencia previa a la Ley 25.871 distinguimos, desde el primer momento, una valorización que varía de empleado a empleado basada en una supuesta dicotomía dentro de la DNM: control versus atención o servicio. Sobre los dispositivos destinados a la atención y solución de trámites para los migrantes, una empleada ( $D N M N^{\circ} 1$ ) evalúa negativamente el funcionamiento de la DNM antes de la Ley 25871:

La ley Videla era tremenda (...) Era dura esa época. Yo llegaba a la oficina, a Migraciones, a las 7:45 AM, allá en Retiro, con el frío que hace en invierno, el calor que hace en verano y veía ya la fila de gente dando la vuelta (...) Era tremendo, porque además era muy difícil obtener una residencia en ese momento y todo eso generaba un montón de irregularidades en el medio. Era horrible. (DNM No 1)

Con una visión opuesta, otro empleado se pregunta, dudando:

¿Lo anterior era bueno? Para mí lo anterior era bueno, tenías posibilidades, no era que no se podía radicar la persona. Estaba más limitadito porque el mismo Estado ejercía su poder de control. Hubo un momento, con el gobierno anterior (de Néstor Kirchner o Cristina Fernández de Kirchner), que se descontroló todo. (DNM No 3, resaltado de la autora)

Además de esta aparente dicotomía entre "controlar bien" o "atender bien", los entrevistados remarcan el carácter "secreto" u "oscuro" que tenía la DNM antes del año 2004. Según DNM N 4, la DNM "era un organismo que, además de ser así medio oscuro, tenía corrupción. (...) hay un montón de cosas que se prestan a eso. Mas con las facultades que tiene la DNM que muchas de ellas son bastantes discrecionales" (DNM No 4). Otra entrevistada relata: "(...) y además había corrupción. (...) Se trata de un organismo complicado, con una densidad... (...)." (DNM N²). El carácter restrictivo de la normativa anterior permitía actos de corrupción y la aparición de:

(...) gente que se servía de esa situación. Era visible, los veías, los sentías, lo comentaban... [eran] apoderados, gestores que te resolvían teóricamente la vía y que en realidad eran miles y miles de documentos falsos que al día de hoy siguen apareciendo. (DNM No 1 )

Aquí observamos que la distancia entre la "ley" y la "realidad" puede aumentar considerablemente debido a la discrecionalidad de los empleados, 
que a su vez es permitida gracias a las características propias de cada gestión y de una estructura organizativa acorde a esas prácticas.

Por otro lado, si el cambio en la "cultura organizacional" implicaba una transformación en el ámbito de las mentalidades de los empleados y funcionarios del organismo, nos preguntamos por las características de esta cultura organizacional previa al año 2004. Para uno de nuestros entrevistados la DNM no tenía una identidad o una "cultura" definida en tanto institución estatal:

(...) la cultura organizacional de la DNM era como un híbrido, un organismo muy cerrado, todo adentro, nada hacia afuera, y no tenía muy claro, los de la DNM, si eran empleados administrativos del Estado o eran una organización cuasi policial. No lo tenían muy claro de hecho creo que estaban más por la opción b que por la a, sobre todo los inspectores. $\left(\mathrm{DNM} \mathrm{N}^{\circ} 4\right)$

Nuevamente advertimos el acento puesto en el control desde una óptica policíaca versus una supuesta vocación de servicio administrativo, ambas presentes en la DNM, haciéndose contrapeso. Como hipótesis, estimamos que la preocupación por el control podría responder a la relación que la DNM mantuvo con las otras fuerzas de seguridad del Estado, observadas por los empleados y funcionarios en algunos detalles, tanto materiales como simbólicos, al interior del organismo. Ahora bien, ¿cómo se establecerían estos vínculos? Según nuestras entrevistas, de diferentes maneras. Una forma directa de relación la encontramos en los cursos de formación de la década del noventa que, según uno de nuestros entrevistados, eran impartidos por integrantes de la Armada Argentina (Fuerza Naval) (DNM N²). Otra forma directa era la ocupación de cargos por parte de militares o ex militares: según este entrevistado, durante los años noventa "muchos de los jefes de área eran capitanes de navío retirados" (DNM N $\left.{ }^{\circ} 2\right)$. Una forma indirecta pero de gran contenido material y simbólico consistiría en, como relató otro entrevistado, los "gorros de la Policía o platitos de Gendarmería" (DNM N²) encontrados a manera decorativa en las oficinas de la DNM de Sede Central. Por último, otra forma indirecta la hallamos en los vínculos intergeneracionales al descubrir, en una misma oficina y en el año 2011, que su Director así como su primera secretaria eran hijos de militares, según otro entrevistados ("la que era mi subjefa, que también era hija de militares", DNM N 5).

\section{Cambios institucionales: resistencias y aceptaciones}

Observamos entonces, según nuestros entrevistados, que a inicios del 2004 primaba dentro de la DNM una identidad institucional más acorde con el control policíaco y que era menester generar acciones destinadas a cambiar esta mentalidad en los empleados y funcionaros. Es decir, no sólo había que modificar los protocolos de atención sino también las actitudes que se correspondían 
con la legislación anterior. Estas acciones fueron, entre otras: capacitación, divulgación interna con contenido identitario (Periódico "Migraciones" de circulación interna), modificaciones en la estructura, vinculaciones con organismos internacionales, académicos, religiosos, de migrantes, etcétera y la "ocupación de espacios" pertenecientes a la problemática de las migraciones en Argentina (foros, ferias, eventos de todo tipo, conflictos políticos, jurídicos y de seguridad).

Estos cambios descriptos, en un organismo de las características de la DNM, generaron resistencias y acomodamientos al interior de cada departamento, Dirección y Delegación. Las resistencias al cambio normativo y de cultura organizacional las encontramos, por un lado, en el trabajo de aquellos empleados que debían "tratar" con los migrantes. Un ex funcionario nos relataba:

[la resistencia a la nueva ley] era muy difícil de ser controlada en el trato habitual del extranjero, había que ejercer una permanente vigilancia para que se aplique la norma, sobre todo cuando ésta no estaba aún reglamentada y había muchas disposiciones que contradecían específicamente el espíritu de la ley. (DNM N²)

Según otro entrevistado, las resistencias en los empleados tienen que ver con cuestiones "culturales" y de mitos sobre los migrantes que no se han logrado desterrar:

Hubo gente que jugó en contra [de los mismos empleados de Migraciones]. Hay aún, gente muy formada en la ley anterior y es difícil el cambio de mentalidad porque es cultural, y la cultura viene de la cuna y eso es muy difícil de transformar (...) empleados de muchos años que trabajaban ahí, el tema de estos mitos de los inmigrantes que nos vienen a robar el trabajo que con los culpables de que nuestros hospitales están mal, que nuestras escuelas estén colapsadas, ese mito está tan fuertemente arraigado. (DNM N ${ }^{\circ}$ 2)

Por otro lado, pese a que los avances tecnológicos lograron el aumento de los controles fronterizos y de permanencia, los rechazos en frontera y las expulsiones, algunos empleados demuestran descontento al considerar que la DNM perdió su poder de policía, como es el caso de $\mathrm{DNM} \mathrm{N}^{\circ} 3$, que expresa:

Bueno, prácticamente la normativa nueva permitía que casi cualquier persona pudiera radicarse, ya no existió más el inhábil absoluto y el inhábil relativo. Yo te diría que hoy, en este momento, quizás una persona, un terrorista internacional, buscado por medio mundo... obviamente que no, pero olvidate de esos y prácticamente se radica todo el mundo. Se pasó del alfa al omega. (DNM No3)

En cuando a las aceptaciones y adaptaciones, una entrevistada refiere a que las resistencias encontradas en algunos empleados no siempre significaban 
un retroceso o un impedimento a los objetivos centrales del organismo, ya que el trabajo se hacía de todas formas tal como se ordenaba. Aun así, las resistencias se hacían evidentes:

La que era mi subjefa, que también era hija de militares [al igual que otros funcionarios en esa oficina], un día hubo una discusión importante dentro del grupo y ella dijo: - yo firmo cosas con las que no estoy de acuerdo todos los días! (...) Pero si, de alguna manera las cosas se hacían, se hacían muy bien y se hacían de acuerdo a lo que decía el Director, le gustara o no les gustara. (DNM N5)

Para finalizar, y teniendo en cuenta las formas de entender al Estado que propusimos en el primer apartado de este artículo, pensamos que los extractos de entrevistas seleccionados demuestran la manera en que los agentes estatales reaccionan a un reacomodamiento de las "reglas del juego", es decir, de la institución misma. Las tensiones originadas por este acontecimiento institucional provenían, sin embargo, de una misma causa: la basculación presente dentro de la DNM entre ser un organismo de control o uno administrativo. Durante el período comprendido entre los años 2004 y 2015 no se abandonaron los dispositivos de control sino que, por el contrario, aumentaron y fueron más eficientes gracias a los avances informáticos. Sin embargo, las acciones destinadas a mejorar el trato, el servicio y a estrechar los vínculos con los migrantes fueron recepcionados, en algunos casos, como un "ablandamiento" del organismo.

Observamos entonces que la pretendida "impersonalidad" del Estado la podríamos encontrar tal vez en la normativa migratoria -si quisiéramos olvidar por un instante que ha sido, ella misma, producto de intensos debates parlamentarios, como explican autores como Badaró (2006), por ejemplopero desaparece al acercarnos para abordar a una institución "desde adentro", donde aparecen las voluntades e intereses de los actores. Habiendo relegado la idea del Estado como "aparato homogéneo de dominación", los primeros resultados de las entrevistas nos permiten hipotetizar que la capacidad de la institución estatal para cristalizar los cambios normativos dependió en muchas ocasiones de la voluntad y de la capacidad de agencia de los actores estatales y, sobre todo, de su margen para hacer frente a los costos políticos de sus decisiones. La ley habilitó y legitimó las transformaciones pero no disolvió las tensiones y los obstáculos internos propios de la estructura estatal de la DNM, que se dan a nivel de los actores y agentes que la constituyen. Y que, finalmente, nos remiten a la manera en la que el Estado ha entendido la cuestión migratoria, pivoteando entre el control y el servicio. Como ejemplo, tomamos la explicación de una ex funcionaria de la DNM que afirma: 
Hubo nuevos organismos, hubo más cooperación interinstitucional con universidades, había publicaciones... una apertura del organismo y algunas resistencias (...) Sí, se pudo gestionar al interior del Estado con la ley de tu lado y un gran margen de autonomía política relativa. Se puede... pero con un gran costo político. (DNM N³)

\section{Conclusiones}

En este artículo nos propusimos examinar, en un punto de inflexión concreto, la manera en que fueron recepcionados al interior de la DNM las acciones llevadas a cabo para redefinir la cuestión migratoria a partir de la Ley de Migraciones $N^{\circ} 25.871$ y lograr que se cristalice a nivel institucional. Este instrumento normativo, debatido y aprobado por el Poder Legislativo, significó la punta del iceberg de la política migratoria: la ley impondría ciertas acciones sucesivas para que la política pudiera implementarse. Sin embargo, nuestra primera revisión normativa indicó que, aunque la nueva ley supuso para el Estado un cambio de orientación política por respectar los DDHH, la misión y función de la DNM como instrumento de gestión no sufrió grandes modificaciones. De hecho, partir del análisis del Informe de Control Interno y Gestión, que abarcaba los años 2003-2007, pudimos observar que la DNM se planteó objetivos que estaban en concordancia con el espíritu de la ley anterior, como el mejoramiento de las funciones de control, tanto de los ingresos y egresos como los de permanencia, y un objetivo general sin especificar: reglamentar de la nueva ley.

Estimamos que fue necesario otro dispositivo normativo, esta vez proveniente del Poder Ejecutivo Nacional, para dar el puntapié hacia el cambio de "las reglas del juego" (Evans, 2007). El Decreto del PEN que declaró en emergencia administrativa a la DNM fijó los lineamientos generales de los problemas y las acciones que debían llevarse a cabo para resolverlos. Pero recién con el cambio de Director General, en el año 2007, se fijaron las acciones que el organismo tuvo que llevar a cabo para poder dar cuenta de las consecuencias inmediatas de la nueva normativa, tanto en su interior como hacia afuera de la institución, presentes en las Memorias Institucionales de Gestión. Allí observamos objetivos que hacían a la capacidad de esta agencia estatal, como por ejemplo la re-estructuración del organismo, optimizar la dotación de recursos humanos (contratación, capacitación y redistribución), modernizar la estructura edilicia y la implementación de sistemas informáticos propios específicos para cada área. Paralelamente, se fueron generando acciones para cambiar "la cultura organizacional": vínculos con migrantes, con organismos de DDHH y académicos, cursos para la atención pública. Coincidimos entonces con lo planteado por Acuña y Chudnovsky (2013) sobre la necesidad de analizar el cambio institucional como una forma de 
administrar y resolver conflictos dentro de una sociedad. Ahora, si invertimos la perspectiva y nos detenemos a observar al Estado "desde adentro", ¿qué sucedería si el mismo cambio institucional genera conflictos al interior del organismo, entre sus empleados y funcionarios?

En la tercera parte de este artículo, las acciones institucionales de la DNM fueron evaluadas a la luz de la experiencia y las vivencias de los funcionarios, exfuncionarios, empleados y exempleados del organismo según dos ejes de análisis: el funcionamiento de la DNM previo al año 2004 y las resistencias a los cambios encontradas u observadas. Desde esta escala de observación, siguiendo a Revel (2015), la "realidad" de la política pública migratoria mostraba sus claroscuros. Según las entrevistas realizadas, la situación de la DNM previa al 2004 mostraba una amplia discrecionalidad de empleados y funcionarios para actuar dentro de la DNM y, consecuentemente, ciertos componentes "secretos", como la corrupción, por ejemplo, que afianzaban la idea de la falta de transparencia estatal. Otra característica de la DNM previa al 2004 era el mal trato y las deficientes condiciones de atención sufridas por los migrantes por parte de los empleados al momento de gestionar sus residencias, que generaba la aparición de gestores o "apoderados", es decir, terceras personas que vulneraban cada vez más la situación del migrante. Notamos, en este primer eje de análisis, una falsa dicotomía entre control al migrante/buena atención al migrante planteada como auto excluyentes.

Sobre el segundo eje, los entrevistados notaron resistencias a los cambios institucionales, especialmente a aquellos destinados a lograr el "cambio cultural" dentro del organismo, es decir, las actitudes y mentalidades enfocadas en el control policíaco hacia el migrante. Esto quiere decir que, para algunos entrevistados, el respeto a los derechos humanos del migrante o el buen trato en el servicio iría en detrimento de la función de control de la DNM, tanto del migrante (permanencia en el país) como de las fronteras. Evidenciamos también ejemplos de vinculación institucional, tanto materiales como simbólicos, de la DNM con diferentes fuerzas de seguridad, observadas por los entrevistados a través de elementos decorativos en algunas oficinas o en los cursos de ingreso impartidos en la década del 90. Pese a estas resistencias, algunos entrevistados manifestaron que éstas no impidieron la correcta aplicación de los cambios emanados de la Dirección Nacional.

A partir de estos dos ejes de análisis pudimos comprobar como el Estado, una vez que nos alejamos de la fría letra de la norma, muestra sus "realidades": un conjunto de prácticas políticas al interior de un organismo destinadas a mantener un sistema de dominación o de poder. Una vez corrido el velo de la idea del "aparato homogéneo de control", esta institución estatal revela sus fisuras y tensiones que giran, generalmente, en torno a la idea del control policíaco del migrante y la del servicio al migrante para su inclusión. Que 
son, en definitiva, dos maneras de entender la cuestión migratoria en tanto cuestión política: la restrictiva y la inclusiva. Por esta razón estimamos que las resistencias al cambio y las valoraciones sobre la situación del organismo previa al 2004 no responden a un simple apego por el statu quo anterior, sino a un modo distinto de comprender su lugar -en términos de podercomo autoridad migratoria. Finalmente, pudimos comprobar que la aparente verticalidad del poder estatal encuentra límites muy concretos en la puesta en práctica de sus decisiones a la hora de penetrar en los diferentes organismos. Las diferencias en las personalidades, las capacidades, las voluntades y apoyos políticos los directores generales de la DNM durante el período analizado pueden servir de hipótesis explicativa de la imposibilidad de aplicar cambios institucionales de envergadura antes del 2008.

En este sentido, consideramos que nuestras primeras entrevistas sugieren algunas líneas de investigación que implicarían, además de la realización de mayor número de entrevistas, un trabajo de campo más exhaustivo indagando en la historia de la DNM y en las vinculaciones - esta vez formales- que tuvo con otras instituciones del Estado, especialmente las fuerzas de seguridad. Es menester, por ejemplo, continuar profundizando en "quién es el Estado", a nivel de las Direcciones Nacionales así como las Direcciones de los principales departamentos, para observar las trayectorias profesionales de quienes ocuparon los cargos de mayor "capacidad de agencia" dentro de la DNM en los últimos 30 años. Si continuamos entendiendo al Estado como relaciones sociales institucionalizadas, conformado y constituido por actores con diferentes intereses y capacidades, estimamos que sólo conoceremos mejor el organismo que detenta la autoridad migratoria en la Argentina si comprendemos quiénes lo conforman, quiénes lo representan -y lo representaron- cotidianamente a través de sus prácticas de interacción con el resto de la sociedad.

\section{Bibliografía}

ABRAMS, Philip. Notas sobre la dificultad de estudiar el estado. Journal of Historical Sociology, v. 1, n. 1, p. 58-89, 1988 [1977].

ACUÑA, Carlos; CHUDNOVSKY, Mariana. Cómo entender las instituciones y su relación con la política: lo bueno, lo malo y lo feo de las instituciones y los institucionalismos. In: ACUÑA, Carlos (comp.). ¿Cuánto importan las instituciones? Gobierno, estado y actores en la política argentina. Buenos Aires: Siglo Veintiuno Editores, 2013, p. 19-67.

ARFUCH, Leonor. Dos variantes del juego de la política en el discurso electoral de 1983. In: VERÓN, Eliseo (ed.). El discurso político: lenguajes y acontecimientos. Buenos Aires: Hachette, 1987.

BADARÓ, Máximo. La conciencia y la ley: la cuestión migratoria en la práctica de las agencias estatales y organismos no gubernamentales en la ciudad de 
Buenos Aires. In: GRIMSON, Alejandro; JELIN, Elizabeth (comps.). Migraciones regionales hacia la Argentina. Diferencia, desigualdad y derechos. Buenos Aires: Prometeo, 2006, p. 207-235.

BOHOSLAVSKY, Ernesto; SOPRANO, Germán (eds.). Un estado con rostro humano: funcionarios e instituciones estatales en Argentina de 1880 hasta la actualidad. Buenos Aires: Prometeo, 2010.

CELS (Centro de Estudios Legales y Sociales). Informe Anual sobre la Situación de los Derechos Humanos en Argentina. Capítulo XIV: A dos años de la nueva Ley de Migraciones: avances, cuestiones pendientes y casos preocupantes. Buenos Aires, 2005.

CORRIGAN, Phillip; SAYER, Derek. The Great Arch: English State Formation As Cultural Revolution. Oxford and New York: Blackwell, 1985.

COURTIS, Corina. Hacia la derogación de la Ley Videla: la migración como tema de labor parlamentaria en la Argentina de la década de 1990. In: GRIMSON, Alejandro; JELIN, Elizabeth (comps.). Migraciones regionales hacia la Argentina. Diferencia, desigualdad y derechos. Buenos Aires: Prometeo, 2006, p. 169-205.

ESPINOZA MOLINA, Ezequiel. Notas sobre la dificultad de etnografiar el Estado. Estudios Sociales del Estado, v. 1, n. 2, p. 175-186, 2015.

EVANS, Peter. Instituciones y desarrollo en la era de la globalización neoliberal. Bogotá: Ilsa, 2007.

LEAL, Alejandra. Burocracia, justicia y pluralismo político. Una exploración de los espacios de poder en Oaxaca. Alteridades, v. 16, n. 31, p. 39-48, 2006.

LINARES, María Dolores. Política migratoria, instituciones y capacidad estatal: la Dirección Nacional de Migraciones (República Argentina) entre los años 2004 y 2015. Polis, n. 48, diciembre 2017. En prensa.

NEJAMKIS, Lucila. Políticas migratorias en la Argentina 1976-2010. De la "Doctrina de Seguridad Nacional" a la consolidación del Derecho Humano a la migración. Buenos Aires: Prometeo, 2016.

NOVICK, Susana. Migración y políticas en Argentina: tres leyes para un país extenso (1876-2004). In: NOVICK, Susana (comp.). Las migraciones en América Latina. Políticas, culturas y estrategias. Buenos Aires: Editorial Catálogos, CLACSO, 2008, p. 131-151.

O'DONNELL, Guillermo; WOLFSON, Leandro. Acerca del Estado, la Democratización y Algunos Problemas Conceptuales. Una perspectiva latinoamericana con referencias a países poscomunistas. Desarrollo Económico, v. XXXIII, n. 130, p. 63-184, 1993.

OSZLAK, Oscar; O’DONNELL, Guillermo. Estado y políticas estatales en América latina: hacia una estrategia de investigación. In: ACUÑA, Carlos (comp.). Lecturas sobre el Estado y las políticas públicas: Retomando el debate de ayer para fortalecer el actual. Buenos Aires, Argentina: Proyecto de Modernización del Estado, Jefatura de Gabinete de Ministros, 2007 [1978]. 
REVEL, Jacques. Juegos de escalas. Experiencias de microanálisis. San Martín: Universidad Nacional de General San Martín, UNSAM EDITA, 2015.

SCHAVELZON, Salvador. La Antropología del estado, su lugar y alguna de sus problemáticas. Publicar, v. VIII, n. IX, p. 72-96, Junio 2010.

SKOCPOL, Theda. El Estado regresa al primer plano: Estrategias de análisis en la investigación actual. In: GROMPONE, Romeo (ed.). Instituciones políticas y sociedad. Lecturas introductorias. Lima: IEP, 1995, p. 92-129.

\section{Entrevistas}

DNM N 1: Empleado, alto funcionario, 16 años antigüedad, Delegación La Pampa. Santa Rosa, año 2014 y 2015. Continúa empleado.

DNM N ${ }^{\circ}$ 2: Empleado, alto funcionario, 10 años antigüedad, Delegación Rosario. Rosario, Marzo 2016. No continúa empleado.

DNM No 3: Empleado, 38 años de antigüedad, Delegación Sede central. Buenos Aires, octubre 2016. Continúa empleado.

DNM N 4: Alto funcionario, 8 años de antigüedad, Delegación Sede Central. Buenos Aires, octubre 2016. No continúa en el cargo.

DNM No 5: Empleado, 3 años de antigüedad, Delegación Sede central. Buenos Aires, octubre 2016. No continúa empleado.

Recibido para publicación en 01.11.2017

Aceptado para publicación en 20.04.2018 Received for publication in November 015t, 2017

Accepted for publication in April 20 $0^{\text {th }}, 2018$

ISSN impresso 1980-8585

ISSN eletrônico 2237-9843

http://dx.doi.org/10.1590/1980-85852503880005312 